\title{
Automated \& Programmable Electron Microscopy Preparation
}

\section{Steven Goodman}

\author{
Microscopy Innovations, LLC, Marshfield, Wisconsin, United States
}

Preparing biological specimens for electron microscopy is tedious, repetitive, often complex, and time consuming. In 2015, Microscopy Innovations introduced the mPrep ASP-1000 Automated Specimen Processor, the first instrument that prepares specimens and grids, and that provides an effectively unlimited number of protocol steps and reagents. The ASP-1000 was designed to meet the needs of small and large labs, to provide ease and speed for routine tasks, and the flexibility for complex protocols including immuno-gold labeling (IGL) and specimen preparation for 3DEM modalities such as serial block face SEM. ASP-1000 protocol programming was intended to enable labs to standardize their methods, create new and custom protocols as needed, and to share protocols with other laboratories. Herein, we review the range of applications and capabilities that have been demonstrated since the ASP1000 introduction.

Briefly, the ASP-1000 is operated by loading tissue or other specimens into mPrep/s capsules, or loading TEM grids (with sections or whole-mount specimens) into $\mathrm{mPrep} / \mathrm{g}$ capsules. The capsules are then attached to the ASP-1000 robotic arm, and the desired protocol is selected or a new protocol is created. Then, under computer control, the protocol is followed by sequentially moving specimen or gridcontaining capsules to reagents arrayed in microtiter plates [1-4].

Some of the breadth of biological TEM and 3DEM applications, developed in collaboration with Microscopy Innovations, is shown in Figures 1-2. This includes the preparation of muscle, kidney, brain, liver, and yeast specimens for TEM (Fig. 1a-e), all prepared "conventionally" using aldehyde fixation, followed by $\mathrm{OsO}_{4}$ post-fix, solvent dehydration, and epoxy embedding. By using continuous agitation, the ASP-1000 can prepare specimens at speeds comparable to microwave processing, as demonstrated by preparation of kidney, yeast, and nerve in $\sim 1 \mathrm{hr}$, and muscle, brain, and liver in $\sim 3 \mathrm{hrs}$, prior to resin polymerization [1-4]. Protocols have also been developed to prepare tissue without $\mathrm{OsO}_{4}$ and with acrylic embedding, as used for immunolabeling (Fig. 1f) [5], and for IGL labeling of tissue sections on grids (Fig. 1g) [6].

In recent years, there has been great interest in 3DEM imaging modalities, as these provide extraordinary 3D ultrastructure information that is not apparent from 2D TEM imaging. However, 3DEM preparation requires more toxic and more complex protocols, typically involving $\mathrm{O}-\mathrm{T}-\mathrm{O}, \mathrm{KFeCN}, \mathrm{Pb}$, and Ur reagents, with manual processing protocols that approach a week [7-9]. With the ASP-1000, 3DEM specimens can be automatically prepared in 24 hours, including resin curing, as demonstrated with brain [7-8], liver [7], and heart right ventricle [9] (Figs. 2a-c).

Many additional protocols have been developed by ASP-1000 users for TEM and 3DEM preparation. These include the preparation of planarian worms in hours, instead of a week, with equivalent results [1012], pre-embedding IGL protocols for brain [13], and post-embedding (on-grid) IGL of bacteria whole mounts [14] and sectioned liver [13], including the convenience of overnight preparation.

In summary, since its introduction in 2015, there are now dozens of programmed specimen and grid protocols presented in publications [1-14], and many more unpublished protocols in use. These protocols are easily shared between ASP-1000 users, and easily modified to create new protocols for new specimen 
types and new applications. This provides a substantial and growing resource library for microscopists seeking the reliability and convenience of automated electron microscopy preparation.
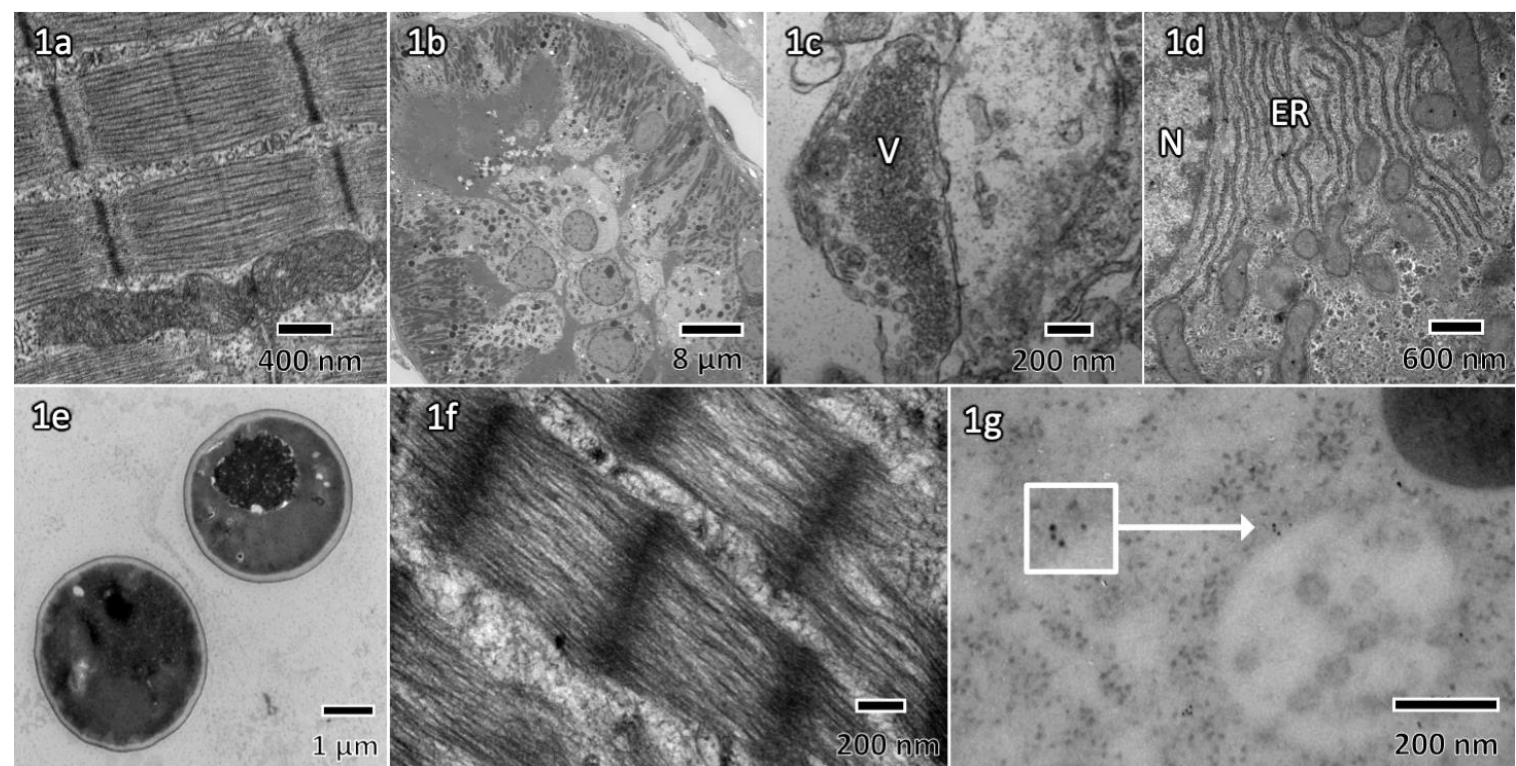

Figure 1. TEM: a) Rat gastrocnemius muscle. b) Mouse kidney. c) Mouse brain, V=synaptic vesicles. d) Rat liver, N=nucleus, ER=endoplasmic reticulum. e) Yeast, agar enrobed. f) Mouse extraocular sarcomeres embedded in LR White resin, no Os postfix. g) IGL-labeled endosomal sorting complex in C. elegans 1-cell embryo.

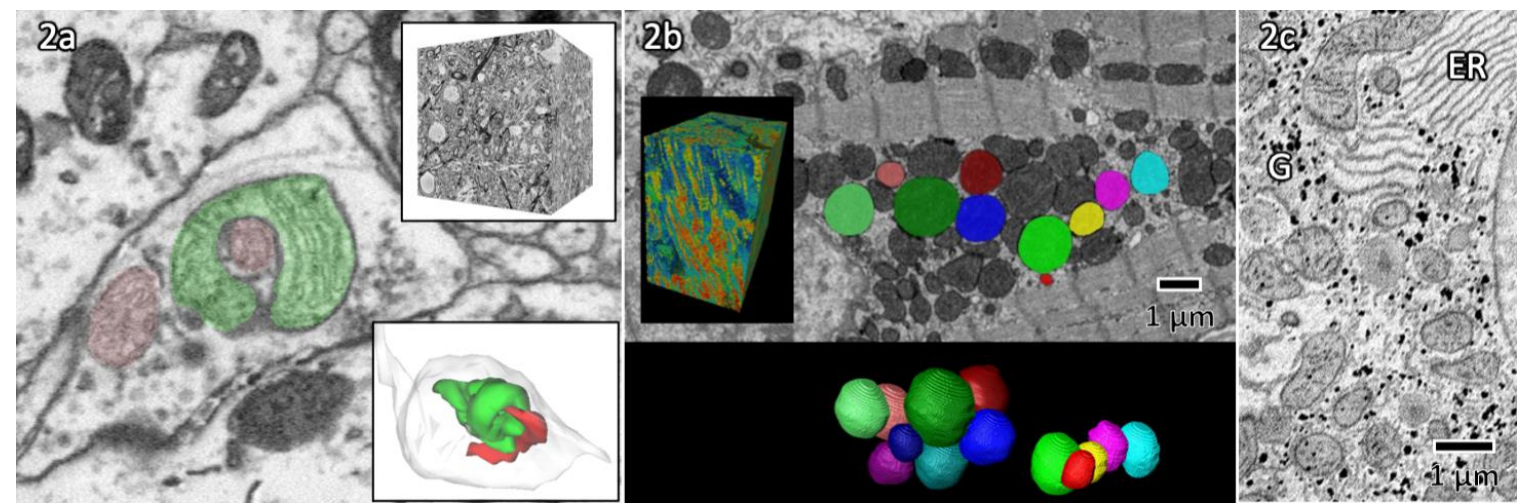

Figure 2. Serial block face SEM. a) Rat brain 3DEM volume, single plane synapse with colored mitochondria, 3D rendering of intertwined mitochondria. b) Rat heart 3DEM volume, single plane with colored mitochondria, 3D rendered mitochondria shapes. c) Rat liver 3DEM single plane image, $\mathrm{G}=$ glycogen granules.

\section{References}

[1] TE Strader et al. Microsc Microanal 2018, 24:1122.

[2] TE Strader et al. Microsc Microanal 2018, 24:1284.

[3] T Strader \& B August. Microsc Microanal 2020, 26:560.

[4] NR Stewart et al. Microsc Microanal 2017, 23:PDP52.

[5] NR Stewart et al. Microsc Microanal 2017, 23:PDP56.

[6] EB Frankel et al. Microsc Microanal 2016, 22:1010.

[7] E Benson et al. Microsc Microanal 2020, 26:534. 
[8] SL Goodman et al. Soc Neuroscience, 2019, 492.02.

[9] SL Goodman et al. Microsc Microanal 2019, 25:PDP46.

[10] M McClain. Microsc Microanal 2016, 22:PDP49.

[11] M McClain et al. Microsc Microanal 2017, 23:PDP59.

[12] M McClain et al. Microsc Microanal 2019, 25:PDP52.

[13] N Marques et al. Microsc Microanal 2018, 24:1300.

[14] EP Lillehoj et al. J Biol Chem 2019, 294(2):662. 